\title{
'Estoy aprendiendo yo con los niños otra vez cómo es el sistema' Modos otros de la experiencia educativa de madres y padres en escuelas chilenas en contextos migratorios*
}

\author{
'I am learning with the children how is the system again' \\ Other modes of the schooling experience of mothers and fathers in Chilean schools \\ in the context of migration
}

\author{
Sara Joiko \\ UCL, Institute of Education. Londres, Reino Unido. \\ sara.joiko.14@ucl.ac.uk
}

\begin{abstract}
RESUMEN
Dado el actual escenario socialmente complejo y educativamente de cambios del campo educativo chileno, sumado a la falta de estudios que visibilicen la experiencia de madres y padres migrantes frente a la educación de sus hijos e hijas; el presente artículo se basa en un estudio cualitativo a partir de entrevistas a padres/madres provenientes de seis países de Latinoamérica. El artículo se centra en la discusión de las estrategias -desde una perspectiva bourdiana- llevadas a cabo por los padres/madres para hacer frente al proceso de ex/inclusión de sus experiencias educativas en un contexto caracterizado por un campo de poder de carácter neoliberal y monocultural. El artículo concluye que las estrategias utilizadas por los padres/madres permiten entender modos otros de la relación familia y escuela que hasta ahora han sido invisibilizados.
\end{abstract}

Palabras claves: Latinoamérica, Bourdieu, interculturalidad crítica.

\begin{abstract}
Given the current socially complex and educationally changing scenario of the Chilean educational field and the lack of studies that have made the experience of migrant mothers and fathers vis-à-vis the education of their children visible; this article is based on a qualitative study interview with parents from six Latin American countries. Moreover, the article focuses on the discussion of the strategies -from a Bourdieusian perspectivecarried out by migrants parents in facing the process of ex/inclusion of their educational experiences in a context characterised by a field of power framed neoliberal and monocultural. The article concludes that the strategies used by parents allow understanding other modes of the family and school relationship that have been invisible until now.
\end{abstract}

Key words: Latin America, Bourdieu, critical interculturality.

\footnotetext{
* Este artículo es parte de mis estudios doctorales gracias al apoyo de CONICYT-Becas Chile N. ${ }^{\circ}$ 72140612. Agradezco encarecidamente a escuelas, municipios y organizaciones sociales; y especial mención a los padres y madres, quienes confiaron en mí y compartieron sus testimonios. Por último, agradezco a la Profesora Carol Vincent y a la Dra. Alice Bradbury por supervisar mi investigación y por sus valiosos comentarios.
} 


\section{MIGRACIÓN, FAMILIA Y EDUCACIÓN}

Durante los últimos años ha habido un aumento en el número de personas migrantes representando hoy un 3,3\% de la población mundial (IOM, 2017). Sin embargo, este fenómeno no es nuevo en el mundo ni menos en Chile. Hoy la migración en el país es de carácter regional dado que el $88 \%$ de la población migrante proviene de países latinoamericanos, representando el 6,6\% de la población nacional (INE \& DEM, 2019). Este porcentaje, aun cuando ha aumentado, se encuentra por debajo del promedio de los países de la OCDE, donde alcanza el 10\% de su población total. Paradójicamente, pese a que compartimos aspectos comunes en términos de idioma, religión, cultura, y un pasado colonial, la sociedad chilena ha percibido la actual migración como un "problema" (INDH, 2017). Según Tijoux, esto se debe principalmente a "su color, su condición, su nacionalidad y su género" (Tijoux, 2016, p. 5). Así mismo, estamos siendo testigos de un racismo institucional por parte de las autoridades políticas quienes en su discurso por una migración “segura, ordenada y regular" (Prensa Presidencial, 2018) están ad portas de aprobar un nuevo proyecto de ley de migraciones que reemplazaría la Ley 1094 (1975), que se aleja de entender la migración como un derecho humano, así como busca imponer "fórmulas" de otros países que nada tienen que ver con la realidad migratoria contemporánea chilena (Thayer, 2019).

A este escenario también se le debe sumar -en directa relación con el presente artículo- la experiencia educativa de padres y madres migrantes. Con experiencia educativa me refiero a cómo los padres/madres migrantes se enfrentan al actual sistema educativo chileno en un escenario socialmente complejo con discursos anti-migración, y los cambios educativos bajo el alero de una Reforma Educacional en curso. Así por ejemplo los padres/ madres migrantes hoy son testigos de la implementación de la Ley de Inclusión Escolar (2015) la que ha declarado explícitamente que el sistema educativo chileno debiese tender a "eliminar todas las formas de discriminación arbitraria" así como "propiciará que los establecimientos educativos sean un lugar de encuentro entre los y las estudiantes de distintas condiciones socioeconómicas, culturales, étnicas, de género, de nacionalidad o de religión" (MINEDUC, 2015, p. 1).

Adicionalmente existen otras dos políticas públicas importantes de señalar en este apartado que complementan el complejo escenario social y educativo en el que padres y madres migrantes se encuentran hoy en día. Antes de finalizar el segundo gobierno de la Presidenta Bachelet se introduce como parte de la reforma una nueva Política de Participación de las Familias y la Comunidad en instituciones educativas (MINEDUC, 2017). Esta política se introduce dado que la relación entre las familias y las escuelas ha sido un desafío no menor en el proceso de la reforma dado que hasta ahora existe una relación ambivalente. Ambivalencia que ha posicionado por un lado a los padres/madres como "consumidores" y por otro como "colaboradores". Por lo tanto, la nueva política de participación ha resaltado la necesidad de generar experiencias de interacción entre escuela, familia y comunidad para crear instancias que se centren en la "tarea de educar para la integración social y la formación ciudadana” (MINEDUC, 2017, p. 18).

En segundo lugar, y con la llegada del segundo mandato presidencial de Piñera el gobierno presentó la Política Nacional de Estudiantes Extranjeros 2018-2022 (MINEDUC, 2018). Esta política fue creada en respuesta a una evaluación institucional llevada a cabo por el gobierno anterior y tiene como objetivo "favorecer el acceso, permanencia 
y trayectorias educativas de estudiantes extranjeros en el sistema educacional chileno, generando dispositivos que den respuesta a las necesidades particulares de este grupo de especial protección" (MINEDUC 2018, p. 24). Sin embargo, esta nueva política no incorpora a las familias, como tampoco hace referencia a la nueva política de participación. En este sentido, dado que el rol de la familia, y más precisamente de padres y madres, todavía se localiza en los márgenes de las políticas estatales. Estudios sobre la relación familia-escuela en contexto migratorio se vuelven necesarios para explorar que sucede en esas interacciones marginadas. Además, si el sistema educativo chileno busca ser inclusivo e intercultural, también debiese ser capaz de identificar las actuales exclusiones que padres/ madres y estudiantes migrantes podrían estar enfrentando.

Por lo tanto, en este escenario socialmente complejo y de cambios educativos, este artículo buscar ser una contribución dado que existe una falta de información sobre la experiencia educativa desde la perspectiva de los padres y madres migrantes con respecto a las escuelas chilenas. Con esto no quiero desconocer la gran cantidad de estudios que se han realizado últimamente en el ámbito educativo que se han enfocado en las dinámicas dentro de las escuelas, por ejemplo, entre estudiantes y profesores así como en las prácticas escolares de inclusión (Bustos \& Gairín, 2017; Hernández, 2016; Jiménez \& Fardella, 2015; Méndez, 2017; Mondaca et al., 2018; Pavez et al., 2018; Poblete et al., 2016; Riedemann \& Stefoni, 2015; Roessler, 2018; Salas et al., 2017; Stefoni et al., 2016;. Tijoux et al., 2018). Sin embargo, existe todavía una ausencia de la mirada desde los padres y madres con respecto al campo educativo chileno. En este sentido el presente artículo se centra en las estrategias de madres y padres migrantes para hacer frente al proceso de ex/inclusión de sus experiencias escolares en un contexto caracterizado por un campo educativo monocultural y neoliberal.

\section{DIALOGANDO CON BOURDIEU Y LA INTERCULTURALIDAD CRÍTICA EN LA RELACIÓN FAMILIA-ESCUELA}

Dado que este artículo se centra en la relación familia-escuela es importante primero dar cuenta de la literatura que críticamente ha estudiado esta relación. En este sentido Vincent $(1996 ; 2000)$ nos propone un marco conceptual sobre las posiciones subjetivas de los padres y madres como una manera de comprender las múltiples formas en que participan dentro y fuera de la escuela. Así mismo, Vincent enfatiza la necesidad de considerar un enfoque interseccional para no homogenizar la categoría de padres/madres, ya que las identidades de estos no pueden ser "reducibles a una sola dimensión" (Crenshaw, 1991 citado por Vincent, 2017), sino que las intersecciones entre clase, género, raza, etnia, nacionalidad (entre otras dimensiones sociales) influyen en las experiencias educativas de padres y madres.

En este sentido, y utilizando el marco conceptual de Vincent, podemos identificar que en Chile la participación de los padres/madres con respecto a la educación de sus hijos/ as se ha estudiado desde la posición que ocupan como consumidores con respecto a la política de elección escolar (Canales et al., 2016; Flores \& Carrasco, 2014; Córdoba, 2014; Elacqua et al., 2006; Gubbins, 2013; Hernández \& Raczynski, 2010; Rojas et al., 2016) y su posición como colaboradores con respecto al apoyo que brindan al aprendizaje escolar (Bustos \& Gairín, 2017b; Gubbins, 2014; Gubbins \& Otero, 2016; Ortega \& Cárcamo, 2018; Ramos, 2017; Reininger \& Santana, 2017; Santana, 2010; Varas \& Núñez, 2016). 
Sin embargo, existen pocos estudios que se han referido a la posición de los padres/ madres como ciudadanos/as con respecto a la educación, y casi inexistentes son aquellos estudios sobre la posicionan que ocupan los padres/madres como fuente de conocimiento de su capital cultural en el contexto escolar (Bellei et al., 2002; Cornejo \& Rosales, 2015; Gubbins, 2001; López et al., 2012; Stefoni et al., 2010; Zamora, 2013).

Sin embargo, las posiciones subjetivas de padres y madres no pueden entenderse por sí solas a nivel individual, sino que también debemos preguntarnos cómo influyen las estructuras que son definidas histórica y políticamente, en la posibilidad de accionar de los agentes. De este modo considero importante dialogar con la teoría de la prácticas sociales de Bourdieu (Bourdieu, 1986; 1990; Bourdieu \& Wacquant, 1992) y la interculturalidad crítica (Diez, 2004; Tubino, 2004; Walsh, 2005) para comprender la relación familiaescuela en contextos migratorios.

A través de su praxeología social, Bourdieu buscaba superar la dicotomía entre objetivismo y subjetivismo de las prácticas sociales, ya que según él la "necesidad estructural y la agencia individual" se refuerzan mutuamente (Bourdieu \& Wacquant, 1992). Para superar esta dicotomía Bourdieu introduce los conceptos de habitus, capital y campo. En este sentido, usando el juego como metáfora, Bourdieu entiende que un campo tiene su "lógica, reglas y regularidades" (Bourdieu \& Wacquant, 1992, p. 104) y los jugadores tienen un conjunto de posiciones desde donde pueden jugar. De manera similar, los agentes hasta cierto punto conocen las reglas y el objetivo del juego, por lo que, cuando entran al campo, posiblemente puedan deducir lo que se espera de ellos/as. Esto es lo que se entiende por habitus como "sistemas de disposiciones duraderas y transponibles" (Bourdieu 1990, p. 53). Aun así, en este juego nada es completamente cierto, dado que Bourdieu no está de acuerdo en que las prácticas sociales sean calculables, como lo enfatiza la teoría de la acción racional, ya que siempre hay un grado de incertidumbre y desinformación.

Por otro lado, la posición que los jugadores/as dependerá de sus capitales (sociales, culturales, económicos y simbólicos) e influirá en la posición que otros puedan tener en el campo. Como en cualquier juego, en el campo de Bourdieu hay movimiento y competencia, lo que muestra que los campos son dinámicos e intercambiables, por lo tanto, un sitio de lucha entre jugadores/as. Sin embargo, una cosa que puede ser diferente de un juego es que la noción de campo de Bourdieu no tiene un perímetro definido, ya que "constituye un espacio de juego potencialmente abierto cuyos límites son fronteras dinámicas" (Bourdieu \& Wacquant, 1992, p. 104). Hasta ahora hemos visto cómo entre los tres conceptos principales de la teoría de Bourdieu existe una complicidad ontológica (Grenfell, 2014), sin embargo, esta complicidad depende del campo de poder, el cual define y legitima las jerarquías del campo en cuestión, en este caso el campo educativo. Es decir, es una lucha "por el principio legítimo de legitimación y por el modo legítimo de reproducción de los fundamentos de la dominación" (Bourdieu y Wacquant, 1992, p. 76). En este sentido, lo que está en juego es el monopolio sobre el poder que se puede utilizar en el campo. Para entender mejor, por lo tanto, este campo de poder que sostengo ha influenciado las experiencias migratorias sur-sur entre las familias migrantes y las escuelas chilenas, creo importante considerar la interculturalidad crítica. Dicha perspectiva que surge del estudio del campo educativo entre académicos/as en América Latina (Diez, 2004; Tubino, 2004; Walsh, 2005), principalmente con el propósito de estudiar la realidad de las comunidades afro descendientes e indígenas; y más recientemente, analizar los movimientos migratorios en la región (Diez, 2013; Novaro, 2012; Riedemann et al., 2018). 
En dicho sentido esta perspectiva es crítica a la hora de entender la forma en que se ha desarrollado la interculturalidad en América Latina. Primero surge como una alternativa al modelo educativo asimilacionista del siglo XX que se convirtió en la "base para la construcción de la homogeneidad requerida por los Estados nacionales modernos" (Ferrão Candau, 2010, p. 335), más adelante, como una opción ante la interculturalidad funcional (Tubino, 2004) bajo un Estado que continuó siendo monocultural y se volvió neoliberal después de las dictaduras latinoamericanas, como en el caso de Chile. La educación y, en consecuencia, las escuelas, se convirtieron en una de las principales herramientas para la reproducción de discursos que diferencian y crean jerarquías étnicas/raciales (Walsh, 2005) en Estados monoculturales de sociedades pluriculturales de Latinoamérica (Tubino, 2004).

De este modo la interculturalidad crítica busca visibilizar que hasta el momento no ha habido una transformación en las relaciones de poder y las estructuras en las sociedades latinoamericanas, que continúan racializando, inferiorizando y deshumanizando (Walsh, 2010) porque son el producto de una matriz social de orden colonial (Walsh, 2004) y un estado monocultural (Tubino, 2005). Así, la propuesta de la interculturalidad crítica es desafiar la visión estrecha de la interculturalidad debido a su naturaleza multidimensional dado que es más que la relación entre culturas, un modelo educativo o una propuesta estatal. Es un "proyecto político-social-epistémico-ético" (Walsh 2010, p. 76) que busca la "incorporación de los tradicionalmente excluidos dentro de las estructuras" (Walsh, 2004, p. 47) así como modos otros de "poder, ser, saber, vivir" (Walsh, 2006).

Es desde estas dos perspectivas en dialogo -entre la interculturalidad crítica en el contexto latinoamericano y la praxeología social de Bourdieu- que este artículo busca preguntarse sobre los modos otros de relación entre las escuelas y las familias en el contexto migratorio y por lo tanto intentar resolver ¿qué sucede cuando padres y madres migrantes se enfrentan a un campo que se regula bajo las lógicas del mercado y en un contexto monocultural? En este sentido, propongo que durante el proceso de ex/inclusión escolar, padres y madres migrantes utilizan diversas estrategias, entendidas estas como prácticas sociales (Bourdieu \& Wacquant, 1992), que al encontrarse con un campo desconocido puede permitir la emergencia de modos otros (Walsh, 2004) de comprender la relación familiaescuela establecida hasta ahora en el campo educativo chileno.

\section{EL ESTUDIO}

El artículo se basa en un trabajo de campo de cuatro meses (mayo a agosto 2016) de una investigación cualitativa exploratoria donde se realizaron entrevistas individuales y grupales con 30 madres y 6 padres migrantes latinoamericanos de ocho escuelas municipales y ocho particulares subvencionadas, de diferentes comunas de la Región Metropolitana (4), Tarapacá (1) y Antofagasta (1). La entrevista fue el método de elección, ya que sitúa las experiencias de los y las participantes en el centro de esta investigación (Lapan et al., 2012). De este modo decidí realizar entrevistas semiestructuradas con el fin de "identificar puntos en común y diferencias entre los entrevistados en uno o más temas" (Lapan et al., 2012, p. 94). Las entrevistas proporcionaron una comprensión profunda de las particularidades, complejidades y significados cotidianos de las prácticas y experiencias escolares de las familias. Las entrevistas fueron transcritas para luego ser analizadas con un enfoque mixto de codificación inductiva y deductiva (Fereday \& Muir-Cochrane, 2006) seguido de un análisis temático (Braun \& Clarke, 2006). 
La mayoría de los padres y madres fueron contactados a través de las escuelas, algunos pocos a través de organizaciones sociales y municipios, y solo tres fueron contactados por el Centro de Padres y Apoderados, lo que era mi intención original ya que este es el espacio formal dentro de las escuelas para padres y madres. De los 36 padres y madres entrevistados, seis de ellos corresponden a padres provenientes de Colombia, Perú y Venezuela. En el caso de las madres a parte de los tres países mencionados también tuve la oportunidad de entrevistar a madres bolivianas, ecuatorianas y dominicanas. Siendo la mayoría de los entrevistados/as de Perú (19) y luego Venezuela (7). En relación a la identificación racial/ étnica, la gran mayoría de los padres y madres no se identificó con ningún grupo (20). Sin embargo, entre aquellos que se refirieron a esta dimensión, la mayoría se identifican como mestizos/as (8), una pareja de padres colombianos como negros, dos madres peruanas se identifican como indígenas, mientras otras dos como blancas, y las dos madres de Ecuador, una se identificó como zamba y la otra mulata. Esta dimensión produjo un interesante debate entre los y las entrevistados dado que la mayoría se identifica fácilmente con una nacionalidad, pero no así con una etnia o raza, aun cuando son relevantes para los procesos de ex/inclusión. Una posible explicación del no reconocimiento de esta dimensión social es que se trata de un proceso de racialización y etnicización en América Latina que es complejo y como indica Walsh (2010) desde la interculturalidad crítica:

La diferencia se construye dentro de una estructura y matriz colonial de poder racializado y jerarquizado, con los blancos y "blanqueados" en la cima y los pueblos indígenas y afro descendientes en los peldaños inferiores (Walsh, 2010, p. 78).

En este sentido, el proceso de no autoidentificación con un grupo étnico/racial puede ser parte de no querer posicionarse en un nivel inferior como establece la matriz colonial, sin embargo, esto no quiere decir que los "otros" no sean identificados en algunos de estos grupos. Este ejemplo de la construcción de la "otredad", en relación con la raza y la etnicidad en el contexto latinoamericano, revela una experiencia explícita de algo implícitamente dicho. Por lo tanto, la experiencia racializada se vive, pero no se nombra. Otro elemento importante de entender con respecto a la raza y etnicidad en la región es el hecho de que personas con la misma nacionalidad no necesariamente se identifican con el mismo grupo. Consideremos por ejemplo el caso de las madres y padres peruanos entrevistados. Diana y Ester ${ }^{1}$ se identificaron como indígenas, mientras que Beti, Marta, Mina, Milly y Nelsy son mestizas y Antonia y Rosario como blancas. Sin embargo, diez padres y madres peruanos no se identificaron con un grupo racial/étnico.

En cuanto a la dimensión que he denominado como ciudadanía, esta se refiere tanto al tiempo vivido en Chile como al tipo de visa. De este modo he distinguido dos grupos. Aquellos padres/madres que se encuentran en un estado temporal, es decir, que llevaban entre un mes y cuatro años residiendo en Chile y se encontraban con una visa sujeta a contrato temporal, o estaban a la espera de alguna de las dos. Del total de madres y padres participantes, 20 se encontraban en este grupo. El segundo grupo de padres/madres que conformaban un total de 16, correspondían aquellos que se encontraban en una situación más permanente dado que llevaban un buen tiempo residiendo en Chile (entre 5 a 20 años) y contaban con una permanencia definitiva. Estoy al tanto que categorizar puede reproducir

Los nombres han sido cambiados para proteger el anonimato de los y las entrevistados a lo largo de este artículo. 
ciertas subjetividades, sin embargo, creo que es importante abordar estos dos tipos de ciudadanía para llamar la atención sobre la influencia -tan invisible hasta ahora- que tienen los regímenes migratorios en términos de derechos en las experiencias educativas de las familias migrantes en Chile. De este modo creo que esta dimensión puede permitir identificar más precisamente las dificultades, tensiones y dilemas que enfrentan cada grupo y que deben ser reconocidas por las políticas públicas, prácticas escolares y la academia.

Una de las dimensiones más complejas de "definir" de la experiencia migratoria es la clase. En el contexto chileno, la clase se basa en variables como los años de escolaridad y los ingresos, pero también abarca una dimensión subjetiva y contextual, lo que Bourdieu identifica como habitus. En el contexto de la migración clasificar a los padres y madres de acuerdo con esta dimensión se vuelve problemático dado que niega la posibilidad de ver la migración como un proceso doble, uno que tiene lugar aquí y allá (Stefoni \& Bonhomme, 2014). En otras palabras, si consideramos la educación y los ingresos, la "clasificación" de clase se vuelve compleja. Algunos padres/madres tienen altos niveles de educación que no son validados como capital cultural en Chile, y esto puede potencialmente obstaculizar sus posibilidades de trabajar y por lo tanto mermar sus ingresos. De este modo, puede resultar en una movilidad descendente. Por ejemplo, algunos padres y madres entrevistados que mencionan tener niveles altos de educación no se encontraban en establecimientos con el mismo grupo socioeconómico de padres/madres ${ }^{2}$. En otras palabras, algunos padres/ madres migrantes tienen niveles más altos de educación en comparación con los padres/ madres "locales" de sus establecimientos.

Finalmente quisiera señalar antes de comenzar el siguiente apartado en donde se presentan las estrategias utilizadas por padres/madres, es que a lo largo del estudio mi rol como investigadora no puede ser ignorado en el sentido de que ciertas tensiones pueden surgir al plantear un estudio que busca visibilizar los procesos de exclusión a los que se ven sujetos actualmente madres y padres migrantes en el contexto chileno. En este sentido, aun cuando la mayoría del tiempo pude percibir una reciprocidad de parte de las familias a hablar sobre los procesos de exclusión a los cuales han tenido que enfrentarse, también hay que tener en cuenta que mi posición como "local" puede haber creado resistencias a hablar de ciertos temas. Por otro lado, estoy segura de que no es que los padres y madres no quieran compartir sus experiencias con quienes hacemos investigación seamos parte o no del país de destino, pero tal vez no ven cómo su participación en un estudio puede generar cambios. Esto creo que es un tema que le corresponde al campo académico plantearse especialmente en estudios de esta naturaleza.

\section{ESTRATEGIAS EDUCATIVAS DE MADRES Y PADRES MIGRANTES}

Yanet, madre venezolana, apoderada de un establecimiento particular subvencionado de la comuna de Santiago que al momento de la entrevista llevaba un año en Chile, cuando al terminar la entrevista le pregunto si pudiera decirle algo a la escuela, ¿qué le diría? Me dice que:

Estoy aprendiendo yo con los niños, otra vez cómo es el sistema.

A lo mejor el colegio tiene fallas, pero yo aún no las noto pues.

Ver nota al pie 1. 
Esta frase resume en gran parte la experiencia escolar de muchas familias migrantes, tanto en Chile como en otras partes del mundo, dado que muchas veces se asume que los sistemas escolares son universales, olvidando que las dinámicas y culturas escolares se han vuelto propias de la constitución de cada nación. De este modo la escuela se ha convertido en el mecanismo por excelencia no solo de una reproducción social, como señalaría Bourdieu en los 70s (Bourdieu, 1973) sino también de lo nacional. Por lo tanto no es raro que cuando los padres/madres migrantes se enfrentan a este "nuevo" campo escolar sientan que están "aprendiendo otra vez cómo es el sistema". "Otra vez" porque no hay que olvidar que las familias traen consigo experiencias escolares de sus países de orígenes. De este modo lo que este artículo busca explorar son las estrategias, desde una perspectiva bourdiana, de los padres y madres entrevistados frente al encuentro con este nuevo campo, las que tienen que ver con acceso, así como hacia la cultura escolar.

\subsection{ACCESO A LA EDUCACIÓN: ¿ELECCIÓN ESCOLAR O EJERCICIO DE UN DERECHO?}

Esta estrategia es el primer encuentro entre padres/madres migrantes y las escuelas chilenas, representando uno de los principales obstáculos mencionados por los y las entrevistados a la hora de enfrentarse al nuevo campo educativo. Recuerdo, más que con cualquier otra pregunta, que al indagar en cómo había sido el proceso de encontrar una escuela, más de un largo suspiro escuché seguida de una larga respuesta de cómo habían vivido el proceso de acceder a la educación en Chile. Así lo vivió Loreto, una madre ecuatoriana que al acercarse a la oficina de ayuda del MINEDUC le indicaron que la búsqueda de escuela era responsabilidad primera y única de los padres/madres:

Incluso el MINEDUC, me acuerdo, que cuando fui a buscar colegio, me dijeron 'no, si usted es la que tiene que buscar colegio', y yo le digo 'pero se supone que yo vengo a la institución, a la sede donde deberían tener una hoja así de grande con todas las direcciones y teléfonos', y me dicen 'no, eso a usted le toca' (Loreto, entrevista).

En este sentido, hay una displicencia del Estado en garantizar el derecho a la educación de todos los niños y niñas independiente de su situación migratoria, situando la responsabilidad únicamente en las "manos" de los padres/madres. De este modo refuerza el rol de los padres/madres como consumidores, lo que en este caso también se mezcla con una sensación de ansiedad entre los padres/madres migrantes recién llegados. Asimismo, a pesar de que la "elección escolar" ha sido situada como la primordial relación entre las familias y las escuelas en contextos de sistemas educativos neoliberales como el chileno, esto estaba lejos de la realidad de los padres/madres entrevistados, quienes principalmente estaban tras la búsqueda de un cupo más que elegir una escuela según la ubicación, recursos económicos, entre otros factores. Por ejemplo, Loreto, quien describe todo este proceso como algo "horrible", al momento de la entrevista era miembro activa de su comunidad y se dedicaba a apoyar a otras familias recién llegadas que tenían que pasar por el proceso de búsqueda de escuela:

Hemos hecho ejercer los derechos en las escuelas, porque hasta ahora en la actualidad si un extranjero viene, pongámosle, el primer semestre, y quieren matricular a su hijo, hay escuelas que son bien cerradas en su metro cuadrado, y no te entienden que no 
depende de uno. Es el sistema de tanto papeleo que no te deja a veces ingresar a los menores a que sigan estudiando. Entonces las escuelas lo que hacen es que te cierran las puertas (Loreto, entrevista).

Además, lo que está en juego en esta estrategia y condiciona el acceso es tanto el capital cultural, así como lo que he denominado el capital cívico. Ambos se manifiestan en "tener los papeles" que se requieren para acceder al campo escolar chileno. En este sentido para acceder a una escuela en Chile (municipal o privada subvencionada), los padres/madres (independientemente de la nacionalidad) tienen que proporcionar diferentes documentos que certifican la identidad del niño (lo que puede entenderse como capital cívico), así como los estudios previos (por lo tanto, el capital cultural). Ambos recursos se convierten en las credenciales en términos de identidad y estudios del estudiante, que permitirán a los padres/ madres inscribir a sus hijos/as en una escuela chilena. Es más, el capital cívico en el campo educativo chileno se materializa en tener un Rol Único Nacional (comúnmente llamado RUT), que es un número de identificación nacional otorgado por el registro civil chileno. Existen diferentes vías para obtener este RUT. En el caso de las familias chilenas y los niños/as de padres/madres migrantes nacidos en Chile, obtendrán este identificador a través de ius solis, lo que significa que toda persona nacida en el territorio chileno tiene derecho a la nacionalidad y por lo tanto un RUT que les permite acceder a servicios públicos, como salud y educación.

En el caso de las familias migrantes, este capital se adquiere a través del padre o madre que posee una visa sujeta a un trabajo que proporciona a los menores de edad un estado migratorio de dependientes. Este proceso otorgaría un estatus de ciudadano/a "similar" a los/as nacionales con respecto a derechos sociales. De este modo se pone en cuestión que aun cuando los niños/as tienen el "derecho" a un cupo en una escuela independiente de la situación migratoria de sus padres/madres, la no posesión del capital cívico a menudo puede contradecir los acuerdos internacionales como la Convención sobre los Derechos del Niño. En este sentido, dado que algunos padres/madres entrevistados no contaban con su situación migratoria al día, se les hacía más difícil acceder a una escuela. Asimismo, a pesar de que algunos padres/madres aún no habían resuelto su visado, ya que estaban en proceso de o acababan de llegar al país, habían logrado inscribir a sus hijos/as en una escuela a través de la solicitud de un número provisorio que hasta hace muy poco se conocía como RUT 100 millones (Joiko \& Vásquez, 2016) y hoy oficialmente tiene el nombre de Identificador Provisorio Escolar (IPE) ${ }^{3}$. A continuación, Armelia - una madre boliviana de una escuela municipal del norte del país- me explica cómo fue el proceso para ella:

(Sara): ¿Qué te pidieron en el colegio?

(Armelia): Los documentos. Hay que traer la libreta legalizada, certificado de nacimiento y una orden de allá, del MINEDUC. Hay que ir a pedir un papel, para que con eso uno pueda dejar inscrito al niño.

(S): ¿Y ahí se acaba?

(A): No se acaba ahí ...necesita tener los documentos, entonces tengo que tramitar los carnet de identidad ... me dan un tiempo para que ahora pueda traerlos ... ahora toca que den unos certificados y luego de que se termine este carnet, que es para un año,

Más información en: https://www.ayudamineduc.cl/ficha/identificador-provisorio-escolar. 
hasta enero, entonces antes de eso tengo que tramitar otra vez para volver a sacar otro ... Es mejor estar legal y no tener problemas.

(S):¿Cómo te has ido informando de los papeles que tienes que hacer?

(A): De a poco he ido preguntando, es que claro, aquí en el colegio fue la cuestión de estudio no más, la libreta y toda la cuestión que tenía que hacer en el MINEDUC. Y lo otro, me he ido informando con otras personas así, los pasos a seguir, fui a la gobernación y ahí me dijeron qué es lo tenía que presentar, y ya presente todo. (Armelia, diálogo).

Sin embargo, a pesar de que los padres/madres entrevistados tuvieron que pasaron por estos procedimientos altamente burocráticos, esto no les impidió encontrar y asegurar un cupo escolar para sus hijos/as. En este sentido, para los padres/madres entrevistados lo que está en juego en esta estrategia es encontrar una vacante más que ejercer su "derecho a la libertad de enseñanza" bajo la forma de la elección. Esto fue mencionado especialmente por los padres/madres recién llegados. De este modo en paralelo a "tener los papeles", comienza un proceso activo de búsqueda de una escuela, y en donde muchas veces los padres/madres se daban cuenta de que no estaban totalmente familiarizados con los procedimientos y que lo que daban por sentado -con respecto a lo que sucedía en sus países de origen- no necesariamente funcionaba en el nuevo campo educativo chileno. Además, en este proceso de búsqueda surge la expresión entre algunos padres/madres de "golpear muchas puertas" para manifestar cómo consiguieron un cupo finalmente para sus hijos/as. Así Vania y Lucio, una pareja peruana recién llegados que usaron la expresión, me cuentan que se acercaron a todas las escuelas cercanas a donde vivían y que incluso habían decidido acercarse al municipio para pedir apoyo. Finalmente terminaron inscribiendo a su hijo en la primera escuela particular subvencionada que les ofreció una vacante, porque en un momento dado se vieron "desesperados":

\section{(Lucio): Llamamos por teléfono}

(Vania): A todos llamamos para pedir cita, pero no había vacantes

(L): Y luego buscamos en internet, y en internet nos apareció otro listado ... y como al $12^{\circ}$ que llamamos... más que todo fue por dejarlo, porque nosotros estábamos ...

(V): Desesperados. (Lucio, diálogo).

La decisión de Vania y Lucio de aceptar el cupo no fue porque era lo que querían en términos de "elección", sino más bien porque estaban preocupados de que su hijo quedara fuera de la escuela. De esta manera la "elección" para los padres/madres entrevistados muchas veces se veía limitada por la forma en que estructuralmente se ofrecen los cupos en el contexto chileno y el viaje migratorio en sí. En términos de la primera ${ }^{4}$-entre las 16 escuelas del estudio- existía una diversidad de rangos de postulación en términos de fechas de inicio y cierre ${ }^{5}$. Por ejemplo, un establecimiento particular subvencionado de la comuna de Santiago solo estipulaba 6 días para el proceso, mientras que un establecimiento

Hoy en día bajo el alero de la Reforma existe un nuevo Sistema de Admisión Escolar (SAE), el que no estaba implementado aún durante el trabajo de campo. Más información en: https://www.sistemadeadmisionescolar.cl.

Información obtenida de la plataforma MINEDUC (http://www.mime.mineduc.cl/mvc/mime/portada), que entrega información autoreportada por los establecimientos y es de uso público. 
particular subvencionado en el norte permitía postular a la escuela durante todo el año. Por otro lado, no existía coherencia en las fechas de postulaciones en establecimientos municipales de la misma comuna. Debemos considerar adicionalmente que estas fechas de postulaciones solo sirven para aquellas familias (independiente de la nacionalidad) que postulan a un cupo escolar para el siguiente año. Además, los padres/madres entrevistados señalan que hay poca claridad en la información por parte de las escuelas y/o del MINEDUC para saber qué sucede con aquellas familias que necesitan matricular a su hijo/a durante el mismo año escolar. Especialmente porque no todas las familias tienen la posibilidad de organizar el movimiento para que coincida con el fin del año escolar en sus países de origen y el comienzo de las escuelas en Chile. De los padres/madres entrevistados esta era una situación común entre venezolanos y dominicanos, dado que, en el caso de los primeros, el calendario escolar va desde a agosto a junio del siguiente año, y en el caso de los segundos, desde septiembre a julio.

Esto fue lo que le sucedió a Wilmer, un padre venezolano recién llegado, que, debido a los cruces de los diferentes calendarios escolares en ambos países, como familia tuvieron que tomar la decisión de migrar separadamente. Lo que significó que él llegó primero parar encontrar trabajo, tener donde vivir y conseguir una escuela, y luego su esposa y dos hijas. También decidieron esperar lo más posible para que sus hijas pudieran terminar adecuadamente el año escolar en Venezuela y así darles "continuidad". Esto significó que su esposa e hijas llegaron en mayo, solo unas semanas antes de que terminara la escuela en Venezuela, sin embargo, casi dos meses más tarde que el comienzo escolar en Chile. Paralelamente al llegar se enfrentaron a otro complejo problema: en el caso de su hija mayor, ella había terminado el equivalente del 1ero básico en Venezuela, por lo tanto, necesitaba comenzar 2do, mientras que la más pequeña necesitaba comenzar kínder. La solución dada por la escuela en donde Wilmer, luego de varios intentos, encontró vacante, fue que ambas tenían que hacer el año escolar nuevamente. Algo que para Wilmer y su esposa fue un poco decepcionante, y como veremos más adelante en la próxima sección, tuvieron que adaptarse para no perjudicar el proceso de aprendizaje de su hija.

Aun cuando para Wilmer la búsqueda de la escuela se transformó en algo complejo, como familia lograron contar con algún tiempo "extra" para organizar el movimiento, lo que en el caso de otros padres/madres entrevistados esto no se dio así dado que la decisión de migrar se realizó en conjunto como familia. Lo que muchas veces significó que buscar una escuela era algo que se debía hacer en paralelo a buscar trabajo, y ambas cosas dependían la una de la otra, lo que es algo no menor si tenemos en cuenta que trabajar es muchas veces una de las principales razones de migrar. Por ejemplo, Eduardo, un padre peruano recién llegado, me explica lo importante que fue para ellos como familia poder encontrar finalmente una escuela:

Yo estoy agradecido, porque vine como desesperado buscando un colegio. Porque yo no quería que mis hijos quedaran sin estudiar este año, porque un año de pérdida es un año, y se atrasa. Igual, yo para trabajar también tenía problemas, o sea no podía trabajar por cuidarlos a ellos, y entonces ahora puedo trabajar por lo que ellos en el día están en el colegio. Y bueno, por el momento estoy agradecido. Y como le vuelvo a repetir, yo andaba desesperado, decía 'un colegio, ¡dónde! ¡dónde!' y tuve la suerte de encontrar acá, y le doy las gracias porque me brindaron el apoyo (Eduardo, entrevista). 
En las palabras de Eduardo se observa la gratitud que los padres/madres sienten cuando al fin alguna escuela les "abren las puertas", algo que más adelante menciono con relación a la dimensión multicultural de la escuela como un factor importante a la hora de "elegir". Este sentimiento de gratitud debe ser observado también críticamente, dado que no podemos olvidar que lo está en juego aquí es un derecho y no un favor caritativo. En este sentido, tanto la decepción de Wilmer y su esposa de tener que ver a sus hijas repetir el año escolar o la desesperación de Eduardo de encontrar escuela para trabajar, no es solo un ejemplo más de la necesaria "desmitificación” de que la estrategia de acceso en el contexto chileno tiene que ver con elección, sino que también resalta la dimensión emocional que este proceso puede tener sobre la experiencia educativa de madres y padres en el contexto migratorio. Dimensión emocional que, especialmente para aquellos padres/madres quienes han visto su migración como parte de un movimiento forzado por los acontecimientos políticos y económicos de sus países de origen, como en el caso de los padres y madres venezolanos, se vuelve más difícil aun cuando en el campo educativo del país de destino, en este caso chileno, el acceso a una escuela está dominado por una narrativa neoliberal de la "elección" en lugar de un derecho y un bien público.

Lo anterior no quita que cuando aparecen algunos elementos que pueden asemejarse a una estrategia de elección como la importancia que los padres/madres le dan a la cercanía de la escuela al hogar o el factor económico, los padres y madres entrevistados se refieren también al factor multicultural que consideran importante para "navegar" el actual campo educativo chileno de carácter monocultural. Por lo tanto, el factor multicultural emerge como parte de una estrategia de los padres y madres para evitar la exclusión, ya que sus hijos/as evitarían ser etiquetados como "los únicos" estudiantes migrantes en la escuela y, por lo tanto, ser vistos como los "otros". También este factor se ha convertido en el eslogan entre las escuelas que las convierte en establecimientos "abiertos" y "acogedores". En este sentido lo multicultural surge como una nueva dimensión en el complejo panorama de la "elección de escuela en Chile" al mismo tiempo que se vincula con la interseccionalidad de la nacionalidad, raza y ciudadanía, lo que posiciona la estrategia de la elección más allá de la clase, como la mayoría de los estudios sobre elección escolar en Chile han determinado.

Lo anterior puede ser observado en el caso de Juan y Sandra, matrimonio colombiano que durante la entrevista me cuentan que desde que llegaron a Chile estaban muy preocupados ya que habían escuchado "que aquí eran muy racistas" con la comunidad negra. Por lo tanto, tomaron la decisión de que para evitar que su hija pasara por algún mal momento en su experiencia escolar, y siguiendo el consejo de un amigo que se encontraba ya en el país, buscaron algún colegio en donde ella no sería la "única", en dicho sentido, que tuviera el factor multicultural. Cuando lograron finalmente el cupo en la escuela que les habían sugerido los "tranquilizó mucho" y reconocen que hubo "buen acogimiento". Además, otros padres y madres entrevistados mencionan que esta dimensión fue importante para el proceso de adaptación de sus hijos/hijas. Para Yanet, era importante que sus hijos compartieran con otros niños/as chilenos, pero también que "sea como multicultural" en el sentido que haya otros niños/as venezolanos. Ella cree que esto es importante para que sus hijos puedan ver que no son los "únicos venezolanos" que emigraron a Chile debido a la situación política del país.

Esta "apertura" de las escuelas muestra una vez más la tensión entre la elección escolar y el ejercicio del derecho a la educación con respecto a la estrategia de acceso de los padres y madres migrantes en el contexto del campo educativo chileno. Resulta problemático 
que los padres/madres se sientan agradecidos de que las escuelas sean "abiertas" cuando, por definición, las escuelas no debiesen ser "puertas cerradas", especialmente aquellas financiadas por el Estado, como lo son los establecimientos municipales y particulares subvencionados. Por lo tanto, garantizar un cupo escolar es un derecho, no un favor para agradecer ni un eslogan que se pueda utilizar para atraer a las familias en el marco de la subvención escolar. Esto último solo sigue reproduciendo la lógica de mercado del campo educativo chileno que hoy se pretende transformar en el contexto de la Reforma Educacional con una Ley de Inclusión Escolar. En este sentido, como hemos visto hasta ahora con esta primera estrategia, no es solo el modelo neoliberal que los padres/madres migrantes tienen que enfrentar con respecto al "nuevo" campo educativo, sino que también al posicionar el factor multicultural en el proceso de acceso se estaría buscando desafiar el carácter monocultural del campo educativo chileno; el que también estaría presente en la siguiente estrategia con respecto al proceso de aprendizaje.

\subsection{AJUSTE HACIA LA CULTURA ESCOLAR: EL AQUÍ Y EL ALLÁ DEL PROCESO DE APRENDIZAJE}

A lo largo de este proceso de ajuste los padres/madres entrevistados mencionan haber tenido que reaprender algunas cosas que daban por sentadas como parte de lo que era la "cultura escolar". Por ejemplo, con relación al horario escolar, las reuniones de apoderados, la organización del aula, el sistema de notas, incluso la comida, entre otras. Algunos padres/ madres incluso mencionan que dado que nadie les explicó cómo funcionaban algunas de estas cosas, a veces se veían implicados en algunos malentendidos con la escuela. En este sentido, uno de los temas más nombrados por los padres/madres con respecto al reaprender esta "nueva" cultura escolar, era el currículo. Tema que tiende a ignorarse como una dimensión relevante en la participación de los padres/madres en las escuelas. De esto modo distinguí dos áreas en particular con respecto a este tema. En primer lugar, los padres/madres se refieren a cómo está organizado el currículum en cuanto a su contenido y en segundo lugar cómo se enseña el mismo. Ambos elementos preocupan a los padres/madres porque para apoyar el aprendizaje de sus hijos/as en su rol como colaboradores se sienten tremendamente excluidos.

Con relación a la organización del plan de estudio, este defiere en cada contexto, dado que el currículo normalmente se organiza nacionalmente según los estándares de cada país. Sin embargo, las escuelas o los padres/madres rara vez reconocen esto. Así, las mismas familias entrevistadas tenían la sensación de que dado que ciertos contenidos en algunas asignaturas se daban después de lo esperado, percibían que en Chile "la educación es más atrasada" en comparación con sus países de origen, lo que incluso influye en la percepción de los padres/madres de la calidad. Por ejemplo. Armelia, me indica que la enseñanza de las multiplicaciones según el currículum chileno se realiza un año más tarde que en Bolivia, y por ello, ella sentía que su hijo "estaba demasiado atrasado". Esto se convierte en un gran desafío para las escuelas, ya que los profesores/as pueden contar en una misma sala de clases con estudiantes que ya conocen parte del contenido y, por lo tanto, los estudiantes se aburren y se desmotivan. Para las familias, por otro lado, este proceso de ajuste al currículum se vincula con el componente emocional antes mencionado del proceso migratorio, ya que pueden sentir que sus hijos/as se están atrasando en su aprendizaje porque ellos como padres/madres han decidido migrar. En el caso del estudio ninguno de los padres/madres menciona que esta decisión es compartida. Antonia, quien ha migrado recientemente desde Perú menciona que esto le preocupa de sobremanera: 
Yo lo estoy pensando, la verdad yo vine a probar suerte acá, tengo una hermana acá. Pero para mí sí es importante lo que es el estudio, y yo siento que al venirnos acá, el nivel de ellos ha bajado, es lo que yo siento. Entonces, si me tuviera que regresar, yo me regreso, me regreso. Y si no, todo mediante Dios que decida, analizaría mucho, analizaría bien qué les conviene estudiar allá o estudiar acá (Antonia, entrevista).

Wilmer, por otro lado, decidió comprarle libros avanzados a su hija, ya que no quería que ella se atrasara con lo que ya había aprendido en Venezuela, dado que -como mencioné antes- su hija tuvo que repetir el año escolar. Por lo tanto, aunque la hija de Wilmer estaba adelantada con respecto a sus pares en la nueva escuela, a él como padre le preocupaba que pudiera aburrirse, ya que la motivación para él es algo crucial para su proceso de aprendizaje. Este dilema "entre la frustración y la adaptación" también se menciona en el estudio reciente de Bustos y Gairín (2017), donde los padres/madres presionan a sus hijos/as para que se desempeñen bien aun cuando la escuela chilena no lo hace.

Con respecto a cómo se enseña, que es el segundo tema destacado con respecto al currículo, los participantes de este estudio mencionan que es algo muy importante para ellos para poder apoyar el proceso de aprendizaje de sus hijos/as y por ende el proceso de adaptación. En este sentido, Mina, una madre peruana, destaca que aun cuando el rol de enseñar es de las escuelas y más precisamente de los profesores/as, si los padres/madres no colaboran no se "puede avanzar". Sin embargo, esta colaboración a veces se hace imposible y algunos padres/madres entrevistados muestran una cierta frustración en este acompañamiento, especialmente en lo que tiene que ver con las tareas. Por ejemplo, Diego, padre colombiano menciona que él ha experimentado esto con la escuela de su hija:

La semana pasada mandaron una tarea a la niña, y a mí me tocó ir a preguntar a los profesores porque no entendí lo que decía. No entendí nada, le dije a mi señora 'no, ¿y ahora cómo hacemos?'. Y yo con vergüenza y todo, vine y le pregunté a la profesora, 'no entendí'. Y ya, entonces, le explicaron a mi señora cómo lo tenía que hacer (Diego, entrevista).

Esta dimensión de la estrategia de ajuste muestra cómo las escuelas asumen que todos los padres/madres conocen el "lenguaje de la escuela", en este caso las instrucciones de las tareas, lo que incluso puede suceder más allá del estado migratorio, por lo tanto, también entre los padres/madres locales. Además, la barrera del lenguaje escolar puede vincularse con la reproducción social en el campo escolar, que está vinculado con un largo debate sobre ¿a quién representa el capital cultural de las escuelas? Este debate ha sido principalmente discutido entre aquellos académicos que han trabajo con el concepto de capital cultural en el contextos de minorías (Rios-Aguilar et al., 2011; Wallace, 2018; Yosso, 2005).

Más allá del lenguaje escolar también está la adscripción nacionalista del currículo. En este sentido, la asignatura de Historia, Geografía y Ciencias Sociales (HGSS) es uno de los temas más difíciles para los padres/madres migrantes como también han señalado con anterioridad los estudios de Alvites \& Jiménez, 2011; Poblete, 2009; Tijoux, 2013. Esto incluso se vuelve contradictorio con el mismo objetivo central de la asignatura que busca que los/as estudiantes: 
Adquieran un sentido de identidad y de pertenencia a la sociedad. Saber quién es, conocer su comunidad y consolidar los lazos con ella son elementos fundamentales para el desarrollo integral de un niño (MINEDUC, 2012, p. 178).

En específico esto se ha convertido en una temática poco resuelta aún entre la educación chilena y las familias peruanas y bolivianas, con respecto a la enseñanza de la Guerra del Pacífico. Este contenido provoca algunos sentimientos nacionalistas de parte de las escuelas chilenas, pero también entre familias peruanas y bolivianas, ya que se espera que vuelvan a "releer" parte de esta historia desde la mirada del chileno. Esto ha sido destacado principalmente por estudios en el norte de Chile donde se produce cierto conflicto y tensión entre las familias y las escuelas (Marín, 2014). Sin embargo, Vania, una madre peruana cuyo hijo va a una escuela privada subvencionada de la Región Metropolitana, también menciona este hecho. Ella me indica que junto a su esposo estaban "aprendiendo la historia de Chile" para apoyar a su hijo, quien no estaba muy interesado en esta materia. Sin embargo, ella termina su relato enfatizando que, aunque los tres, madre, padre e hijo, se sentaban "a leer lo que es la Historia de Chile" aunque su hijo todavía era "súper peruano". De su relato pareciera surgir la posibilidad de que, al asimilar la historia de Chile, su hijo podría convertirse de inmediato en chileno y anti peruano, lo que puede ser un signo de cómo la historia de esta guerra ha sido representada por las escuelas chilenas.

Loreto, por otro lado, fue mucho más crítica con lo que estaba sucediendo con respecto a los contenidos que se enseñan en la escuela, a pesar de que la escuela pública de sus hijas es reconocida como una de las escuelas en el norte del país con una importante matrícula de estudiantes migrantes (50\% según las estadísticas del MINDUC) y ha estado trabajando con algunas intervenciones para fortalecer el enfoque intercultural dentro del proyecto educativo del establecimiento (Marín, 2016). Loreto, sin embargo, aún sentía que el currículo estaba demasiado orientado hacia Chile y que poco espacio se le otorgaba al conocimiento de otras culturas:

Chile es el único país que hasta ahora creo que conozco, que enseña lo de Chile. No está tan familiarizado con las cosas del exterior, es todo de Chile. Eso no ocurre en los otros países, te enseñan más de las otras culturas y la tuya también (...) Entonces eso también les cuesta harto a los niños. Matemáticas va a ser matemáticas a nivel mundial, lenguaje igual, pero sociales, historia, todo eso, geográficamente se enseña más lo de aquí (Loreto, entrevista).

Una posible hipótesis de por qué a pesar de la política interna de la escuela por trabajar bajo un enfoque intercultural este no se da y es ignorado a la hora de enseñar, es que el currículo tiene directa relación con los estándares nacionales, por lo tanto, la escuela se vería limita a ajustar y flexibilizar las materias dado que son "evaluadas" a través del SIMCE. Esto contradeciría lo que se ha denominado como la aceptabilidad (Tomaševski, 2009) del derecho a la educación, lo que significa que se espera que los sistemas educativos adapten sus currículum para que sean pertinentes a la realidad de las escuelas. Por lo tanto, el campo educativo chileno no ha podido satisfacer las necesidades y las demandas del nuevo contexto, y ha perpetuado un sistema de educación monocultural. Para superar esta invisibilidad de la diversidad en la escuela, Loreto, como miembro activa de su comunidad ecuatoriana, sugirió llevar algunas actividades culturales a la escuela, lo que fue bien 
recibido por el director, pero que podemos observar se posicionan fuera del aula, más bien como parte de actividades extracurriculares, las que se han comenzado a volver una práctica común a través de las "celebraciones/encuentros multiculturales".

Aun cuando estos nuevos espacios de participación se encuentran fuera del aula, se han convertido en un importante vínculo entre las familias y la escuela, ya que las escuelas que no tienen el conocimiento de cada uno de los países que compone esta nueva "escuela multicultural", requieren de la participación de las familias para abordar estas identidades diversas, convirtiéndose así en una importante fuente de conocimiento de su capital cultural. Estas celebraciones pueden involucrar la presentación de diferentes manifestaciones culturales de cada país o se realizan en base a la celebración del día de la independencia. Para Ester, madre peruana de una escuela municipal con enfoque intercultural de la región metropolitana, estos espacios de participación son muy importantes para intercambiar experiencias:

Entonces todo eso genera un espacio que la mayor parte de la comunidad que vive acá le gusta ¿a quién no le va a gustar escuchar la música de su país y que la gente participe y se integre? Espacios donde uno pueda contar su experiencia (Ester, entrevista).

A pesar de que los encuentros multiculturales o la celebración del día de la independencia de cada país pueden quedar enmarcados desde un enfoque nacionalista, para Ester y otros padres/madres migrantes entrevistados, son espacios importantes para el reconocimiento de las múltiples identidades que conforman la escuela chilena hoy, que durante muchos años han sido silenciadas y marginadas bajo una mirada monocultural del campo educativo chileno. En dicho sentido, posicionar a los padres y madres como una fuente de conocimiento de su capital cultural se vuelve crucial para un proceso emergente de decolonizar el currículum y la cultura escolar.

\section{DISCUSIÓN FINAL}

Volviendo al objetivo central del artículo de describir las estrategias de madres y padres migrantes para hacer frente al proceso de ex/inclusión de sus experiencias escolares en un contexto caracterizado por un campo educativo monocultural y neoliberal, podemos decir que en el caso de la estrategia de acceso, como el primer encuentro entre padres/ madres migrantes, estos tuvieron que aprender a jugar el juego del mercado y asumir un rol de consumidores, dado que para el Estado son ellos/as los primeros y últimos responsables de la educación de sus hijos/as. De este modo dado que el proceso era complejo, confuso y a menudo emocional, las escuelas como una extensión del Estado, en lugar de responsabilizarse, se convertían en "almas caritativas" al recibir con las "puertas abiertas" a las familias migrantes, por lo que los padres/madres se muestran agradecidos por permitirles ingresar. Además, esto ha tenido un efecto en la percepción de los padres/ madres con respecto a la importancia del factor multicultural a la hora de buscar una escuela, ya que proporciona una herramienta de resistencia a la discriminación y a su vez de ser posicionados como los otros en una educación monocultural. Además, queda expuesto como el capital cívico, el que se manifiesta en contar con los "papeles necesarios" pueden determinar cuán fácil/difícil es acceder a la escuela, lo que se convierte a todas luces en una restricción hacia la "elección" final. Por lo mismo, esta estrategia ilustra que lo que está en 
juego es un derecho más que una elección, de este modo la posición de los padres/madres como ciudadanos/as se pone en tensión con un rol ilusorio de consumidor.

Con relación a la segunda estrategia abordada, se puede observar cómo ciertas frustraciones de los padres/madres migrantes emergen, especialmente cuando comparan la experiencia escolar de sus hijos/as en Chile y sus países de origen, que son frustraciones que a veces se fundamentan en malentendidos. Con esta estrategia de ajuste no pretendo hacer hincapié en un proceso de asimilación llevado a cabo por los padres y madres migrantes hacia el campo educativo chileno. Al contrario, creo importante enfatizar en las formas en que es posible que puedan surgir modos otros de la relación entre la familia y la escuela cuando existe una "discrepancia entre habitus y campo" (Bourdieu y Wacquant, 1992, p. 130). Desde este punto de "incomodidad" es donde surgen problemas como las tensiones que se observan en esta estrategia con respecto a la calidad de la educación o el currículum. Sin embargo, "cuando el habitus encuentra un campo con el que no está familiarizado, la disyunción resultante puede generar cambio y transformación" (Reay, 2004, p. 436). En este sentido, esta frustración se puede superar al encontrar otros modos de relacionarse con las escuelas de sus hijos/as, como en el caso de Wilmer, quien compra libros avanzados para su hija o como Loreto cuando le propone a la escuela la creación de espacios culturales. También es importante observar como a partir de esta "incomodidad" los padres/madres utilizan su capital cultural -frecuentemente marginado- para hacer frente a un campo educativo monocultural que constantemente niega la diversidad de sus aulas de clases. De este modo, la mirada de los padres/madres migrantes sobre el campo educativo chileno se vuelven un buen ejercicio que refleja las tensiones propias del sistema escolar chileno en la relación familia-escuela más allá del contexto de la migración.

Finalmente, y dado que a veces la academia olvida cuál es el propósito final de producir conocimiento para transformar los procesos de exclusión existentes, quisiera terminar con algunas recomendaciones que surgen a partir de lo aquí señalado. En primer lugar, creo importante seguir insistiendo, como lo han hecho diversas campañas desde organizaciones sociales, que el Estado independiente de la situación migratoria de los padres/madres, debiese asegurar la entregar de un RUN definitivo a cada niño, niña y adolescente. El actual IPE, todavía no asegura una completa inserción al sistema educativo y el ejercicio pleno de derechos sociales. En segundo lugar, el proceso de postulación a las escuelas debiese estar centralizado y transparentado, existiendo a su vez información pública que explicite los procedimientos para postular fuera de las fechas 'oficiales', por ejemplo, en el caso de llegar después del comienzo escolar. Este proceso, el que es el primer encuentro entre las familias y las escuelas tiene que dejar de ser una traba. Tercero, es importante derribar mitos con respecto a la escuela, es decir, se debe entregar información clara a los padres y madres para que conozcan cómo se enseñan las materias, cómo se organiza el currículum, así como cualquier otro tipo de información relevante para transformar la experiencia escolar de padres y madres migrantes en un proceso de inclusión antes que en un proceso de exclusión.

\section{REFERENCIAS BIBLIOGRÁFICAS}

Alvites, L. \& Jiménez, R. (2011). Niños y niñas migrantes, desafío pendiente. Innovación educativa en escuela de Santiago de Chile. Synergies Chili, (7), 121-136.

Bellei, C., Gubbins, V. \& López, V. (2002). Participación de los centros de padres en la educación. 
Estudios Pedagógicos XLV, N 3: 93-113, 2019

'ESTOY APRENDIENDO YO CON LOS NIÑOS OTRA VEZ CÓMO ES EL SISTEMA' MODOS OTROS DE LA EXPERIENCIA EDUCATIVA DE MADRES Y PADRES EN ESCUELAS CHILENAS EN CONTEXTOS MIGRATORIOS

Expectativas, demandas, desafíos y compromisos. Santiago: UNICEF. Disponible en: http:// www.unicef.cl/archivos_documento/30/Centro_Padres1.pdf.

Bourdieu, P. (1973). Cultural reproduction and social reproduction. En R. Brown (Ed.), Knowledge, education and cultural change: papers in the sociology of education [contributed to the Annual Conference of the British Sociological Association held at the University of Durham, 7-10 april 1970].

. (1986). The forms of capital. En J. G. Richardson (Ed.), Handbook of Theory and Research for the Sociology of Education (pp. 241-258). New York: Greenwood Press.

(1990). The Logic of Practice. California: Stanford University Press.

Bourdieu, P. \& Wacquant, L. (1992). An invitation to reflexive sociology. Chicago: University of Chicago Press.

Braun, V. \& Clarke, V. (2006). Using thematic analysis in psychology. Qualitative Research in Psychology, 3(2), 77-101.

Bustos, R. \& Gairín, J. (2017). Expectativas académicas de estudiantes y padres migrantes- el caso de Arica en la frontera de Chile y Perú. Revista Dilemas Contemporáneos: Educación, Política $y$ Valores, 4(3), 1-28.

Canales, M., Bellei, C. \& Orellana, V. (2016). ¿Por qué elegir una escuela privada subvencionada? Sectores medios emergentes y elección de escuela en un sistema de mercado. Estudios Pedagógicos, XLII(3), 89-109.

Córdoba, C. (2014). La elección de escuela en sectores pobres: Resultados de un estudio cualitativo. Psicoperspectivas. Individuo y Sociedad, 13(1), 56-67.

Cornejo, R. \& Rosales, A. (2015). Objective Structures and Symbolic Violence in the Immigrant Family and School Relationships: Study of Two Cases in Chile. Social Science, 4(4), 1243-1268.

Diez, M. L. (2004). Reflexiones en torno a la interculturalidad. Cuadernos de Antropología Social, 147(19), 191-213.

(2013). Repensando la interculturalidad en educación: aportes de la investigación socioantropológica a un campo problemático. Revista Docencia, 51, 4-17.

Elacqua, G., Schneider, M. \& Buckley, J. (2006). School choice in Chile: Is it class or the classroom? Journal of Policy Analysis and Management, 25(3), 577-601.

Fereday, J. \& Muir-Cochrane, E. (2006). Demonstrating Rigor Using Thematic Analysis: A Hybrid Approach of Inductive and Deductive Coding and Theme Development. International Journal of Qualitative Methods, 5(1), 80-92.

Ferrão Candau, V. M. (2010). Educación Intercultural En América Latina: Distintas Concepciones y Tensiones Actuales. Estudios Pedagógicos, XXXVI(2), 333-342.

Flores, C. \& Carrasco, A. (2014). Choosing schools: parental preferences, constraints to school choice and school segregation in Chile. Tercer Congreso Interdisciplinario de Investigación en Educación. 21 y 22 de agosto 2014. Hotel Crowne Plaza Santiago, Chile.

Grenfell, M. J. (Ed.). (2014). Bourdieu: key concepts. Routledge.

Gubbins, V. (2001). Relación entre escuelas, familias y comunidad: Estado presente y desafío pendientes. De Familias y Terapias, 9, 107-123.

. (2013). La Experiencia subjetiva del proceso de elección de establecimiento educacional en apoderados de escuelas municipales de la Región Metropolitana: a subjective experience. Estudios Pedagógicos, $X X X I X(2), 165-178$.

(2014). Estrategias educativas de familias de clase alta. Revista Mexicana de Investigacion Educativa, 19(63), 1069-1089.

Gubbins, V. \& Otero, G. (2016). Efecto de la rendición de cuentas de las escuelas chilenas sobre la participación de padres. Revista de Educación, (372), 9-34.

Hernández, A. (2016). El currículo en contextos de estudiantes migrantes: Las complejidades del desarrollo curricular desde la perspectiva de los docentes de aula. Estudios Pedagógicos XLII(2), $151-169$. 
Hernández, M. \& Raczynski, D. (2010). Cómo eligen escuela las familias chilenas de estratos medios y bajos? Información, representaciones sociales y segregación. Disponible en: http:// www.ciie2010.cl/docs/doc/ sesiones/211_DRaczynski_Eleccion_de_escuela_informacion.pdf.

INDH. (2017). Informe Anual. Situación de los Derechos Humanos en Chile. Santiago: INDH. Disponible en: https://www.indh.cl/bb/wp-content/uploads/2017/12/01_Informe-Anual-2017. pdf.

INE \& DEM. (2019). Estimación de personas extranjeras residentes en Chile. Disponible en: https:// www.extranjeria.gob.cl/media/2019/02/Presentación-Extranjeros-Residentes-en-Chile.-31Diciembre-2018.pdf.

IOM. (2017). World Migration Report 2018. Disponible en: https://publications.iom.int/system/files/ pdf/wmr_2018_en.pdf.

Jiménez, F. \& Fardella, C. (2015). Diversidad y rol de la escuela: discursos del profesorado en contextos educativos multiculturales en clave migratoria. Revista mexicana de investigación educativa, 20(65), 419-441.

Joiko, S. \& Vásquez, A. (2016). Acceso y elección escolar de familias migrantes en Chile: "No tuve problemas porque la escuela es abierta, porque acepta muchas nacionalidades". Calidad de la educación, (45), 132-173.

Lapan, S., Quartaroli, M. \& Riemer, F. (2012). Qualitative research: An introduction to methods and designs. San Francisco: Jossey-Bass.

López, V., Madrid, R. \& Sisto, V. (2012). Red light in Chile: Parents participating as consumers of education under global neoliberal policies. Globalization-Education and Management Agendas, 28-54.

Marín, J. (2014). Hijos/as de inmigrantes en el norte de Chile: Hacia la construcción de una escuela intercultural. Santiago de Chile: Haz tu tesis en cultura. Disponible en: http://www. observatoriocultural.gob.cl/wpcontent/ uploads/2015/05/tesis_jair_marin.pdf.

Marín, J. (coord.), (2016). Manual la infancia mundial en la escuela aumenta el capital cultural. Iquique: Fosis.

Méndez, L. (2017). Niñez y migración en Antofagasta, Chile: relatos sobre racismo e interculturalidad. En M. García Barrera \& F. Maniglio (Eds.), Los Territorios Discursivos de América Latina Interculturalidad, Comunicación e Identidad. Quito: CIESPAL.

MINEDUC. (2012). Bases curriculares Educación Básica. Santiago de Chile. Disponible en: http://archivos.agenciaeducacion.cl/biblioteca_digital_historica/orientacion/2012/bases_ curricularesbasica_2012.pdf.

MINEDUC. (2015). Ley de Inclusión Escolar. Disponible en: https://www.leychile.cl/ Navegar?idNorma $=1078172$.

MINEDUC. (2017). Política de participación de las familias y la comunidad en instituciones educativas. Disponible en: http://basica.mineduc.cl/wp-content/uploads/sites/25/2017/04/ Política-de-Participación-de-la-Familia-y-la-Comunidad-en-instituciones-educativas.pdf.

MINEDUC. (2018). Política Nacional de estudiantes extranjeros 2018-2022. Disponible en: https:// migrantes.mineduc.cl/wp-content/uploads/sites/88/2018/06/POLITICA-NACIONAL-EEFinal-1-1.pdf.

Mondaca, C., Gairín, J., \& Muñoz, W. (2018). Estudiantes migrantes peruanos en el sistema educativo de la región de Arica y Parinacota, norte de Chile. Interciencia, 43(1), 28-35.

Novaro, G. (2012). Niños inmigrantes en argentina. Nacionalismo escolar, derechos educativos y experiencias de alteridad. RMIE, 17(53), 459-483.

Ortega Arias, M. D. \& Cárcamo Vásquez, H. (2018). Relación familia-escuela en el contexto rural. Miradas desde las familias. Educación, XXVII(52), 98-118.

Pavez, I., Ortiz López, J. E., Jara, P., Olguín, C. \& Domaica, A. (2018). Infancia haitiana migrante en Chile: barreras y oportunidades en el proceso de escolarización. Entre Diversidades, (11), 71-97. 
Estudios Pedagógicos XLV, $\mathrm{N}^{\circ}$ 3: 93-113, 2019

'ESTOY APRENDIENDO YO CON LOS NIÑOS OTRA VEZ CÓMO ES EL SISTEMA' MODOS OTROS DE LA EXPERIENCIA EDUCATIVA DE MADRES Y PADRES EN ESCUELAS CHILENAS EN CONTEXTOS MIGRATORIOS

Poblete, R. (2009). Educación Intercultural en la escuela de hoy: reformas y desafíos para su implementación. Revista Latinoamericana de Educación Inclusiva, 3(2), 140-156.

Poblete, R., Galaz, C., Alvarez, C., Berrera, M. F., Hedrera, L. \& Olivares, Y. (2016). Niños y niñas inmigrantes: Trayectorias de inclusión educativa en escuelas de la Región Metropolitana. Santiago: MINEDUC.

Prensa Presidencial. (2018). Presidente Piñera presenta reforma para "garantizar una migración segura, ordenada y regular". Disponible en: https://prensa.presidencia.cl/comunicado.aspx?id=73015

Ramos M. (2017). Hows and whys of parental involvement in a national 'neoliberal laboratory': aspirations, values and beliefs in relation to children's education among Chilean urban lowermiddle-class parents. Compare: A Journal of Comparative and International Education, 1-17.

Reay, D. (2004). 'It's all becoming a habitus'- Beyond the habitual use of habitus in educational research. British Journal of Sociology of Education, 25(4), 431-444.

Reininger, T. \& Santana, A. (2017). Parental involvement in municipal schools in Chile: Why do parents choose to get involved? School Psychology International, 38(4), 363-379.

Riedemann, A., \& Stefoni, C. (2015). Sobre el racismo, su negación, y las consecuencias para una educación anti-racista en la enseñanza secundaria chilena. Polis. Revista Latinoamericana, (42).

Riedemann, A., Stefoni, C., Stang, F. \& Corvalán, J. (2018). Desde una educación intercultural para pueblos indígenas hacia otra pertinente al contexto migratorio actual. Un análisis basado en el caso de Chile. Estudios Atacameños, Arqueología y Antropología Surandinas.

Rios-Aguilar, C., Kiyama, J. M., Gravitt, M. \& Moll, L. C. (2011). Funds of knowledge for the poor and forms of capital for the rich? A capital approach to examining funds of knowledge. Theory and Research in Education, 9(2), 163-184.

Roessler, P. (2018). Pensamiento nacionalista-territorializado y percepción de "des-ubicamiento" del inmigrante: el camino hostil de las construcciones de identidades chilenas en la convivencia escolar. Calidad de la educación, (49), 50-81.

Rojas, M. T., Falabella, A. \& Leyton, D. (2016). Madres de clases medias frente al mercado educativo en Chile: decisiones y dilemas. En J. Corvalán, A. Carrasco, \& J. E. García Huidobro (Eds.), Mercado Escolar y Oportunidad Educacional: Libertad, Diversidad y Desigualdad. Santiago: Ediciones UC.

Salas, N., Kong, F. \& Gazmuri, R. (2017). La investigación socio territorial: Una propuesta para comprender los procesos de inclusión de los migrantes en las escuelas. Revista latinoamericana de educación inclusiva, 11(1), 73-91.

Santana, A. (2010). Relación familia escuela en contextos de pobreza. Posibilidades y limitaciones en los procesos educativos. Revista CUHSO, 20(2), 81-93.

Stefoni, C., Acosta, E., Gaymer, M. \& Casas-Cordero, F. (2010). El derecho a la educación de los niños y niñas inmigrantes en Chile. Bilbao: Universidad de Deusto.

Stefoni, C. \& Bonhomme, M. (2014). Una vida en Chile y seguir siendo extranjeros. Revista de Estudios Transfronterizos, XIV(2), 81-101.

Stefoni, C., Stang, F. \& Riedemann, A. (2016). Educación e interculturalidad en Chile: un marco para el análisis. Estudios Internacionales, (185), 153-182.

Thayer, L. E. (2019). Entrevistado por Fernando Paulsen, 17 de enero 2019. Disponible en: https:// www.cnnchile.com/lodijeronencnn/ley-de-migraciones-sociologo-luis-eduardo-thayer-criticola-inexistencia-de-formulas-que-permitan-aplicar-este-proyecto_20190117/?fbclid=IwAR02m3VMd-2E11lh_jHGWmezq5SdeS3qcU77BI1RSFrukRZMy63OYMxD6Y.

Tijoux, M. E. (2013). Las escuelas de la inmigración en la ciudad de Santiago: Elementos para una educación contra el racismo. Polis, Revista Latinoamericana, 12(35), 287-307.

(2016). El cuerpo como cicatriz. Relaciones coloniales y violencia racista.

Tijoux, M. E., Peyrau, G., Marín, J. \& Palma, F. (2018). Afectos y efectos de una educación intercultural y antirracista. Experiencia en torno a la migración del Colegio República de Croacia de Iquique. Santiago: Comunicaciones y Gráfica Limitada S.A. 
Tomaševski, K. (2009). Human rights obligations: making education available, accessible, acceptable and adaptable. Disponible en: https://www.right-to-education.org/sites/right-to-education.org/ files/resource-attachments/Tomasevski_Primer\%203.pdf.

Tubino, F. (2004). Del interculturalismo funcional al interculturalismo crítico. En M. Samaniego \& C. Garbarini (Eds.), Rostros y fronteras de la identidad (pp. 151-164). Temuco: Universidad Católica de Temuco.

Varas, M. \& Núñez, T. (2016). Teorías subjetivas de los apoderados de un colegio particular subvencionado de Antofagasta respecto a su participación en el ámbito académico de sus hijos. Estudios Pedagógicos XLII(2), 227-245.

Vincent, C. (1996). Parents and Teachers: Power and Participation. London: Falmer Press. . (2000). Including parents?: education, citizenship and parental agency. Birmingham: Open University Press.

. (2017). 'The children have only got one education and you have to make sure it's a good one': parenting and parent-school relations in a neoliberal age. Gender and Education, 29(5), 541-557.

Wallace, D. (2018). Cultural capital as whiteness ? Examining logics of ethno-racial representation and resistance. British Journal of Sociology of Education, 39(4), 466-482.

Walsh, C. (2004). Interculturalidad crítica y pedagogía de-colonial: Apuestas (des)de el in-surgir, re-existir y re-vivir. . (2005). Interculturalidad, conocimientos y decolonialidad. Signo y Pensamiento, 46(24), $40-50$.

. (2006). Interculturalidad y (de)colonialidad: diferencia y nación de otro modo. Desarrollo e interculturalidad, imaginario y diferencia: La nación en el mundo andino, 27-43.

. (2010). Interculturalidad crítica y educación intercultural. En Construyendo Interculturalidad Crítica (pp. 75-96). La Paz, Bolivia: Instituto Internacional de Integración del Convenio Andrés Bello.

Yosso, T. J. (2005). Whose culture has capital? A critical race theory discussion of community cultural wealth. Race Ethnicity and Education, 8(1), 69-91.

Zamora, G. (2013). La democracia y la participación en la escuela: ¿cuánto se ha avanzado desde las normativas para promover la participación escolar? Revista de Estudios y Experiencias en Educación, 10(9), 107-129. 
